\title{
Effect of Particle Size in the TL Response of Natural Quartz Sensitized by High Dose of Gamma Radiation and Heat-Treatments
}

\author{
Álvaro Barbosa de Carvalho Jr. ${ }^{\mathrm{a}}$, Pedro Luiz Guzzo ${ }^{\mathrm{b}, *}$, Henry Lavalle Sullasic $^{\mathrm{c}}$,Helen Jamil Khoury ${ }^{\mathrm{d}}$ \\ ${ }^{\mathrm{a}}$ Graduate Program of Mechanical Engineering, Federal University of Pernambuco, \\ CEP 50740-530 Recife, PE, Brazil \\ ${ }^{\mathrm{b}}$ Department of Mining Engineering, Federal University of Pernambuco, \\ CEP 50740-530 Recife, PE, Brazil \\ ${ }^{\mathrm{c}}$ Department of Archeology, Federal University of Pernambuco, \\ CEP 50670-900 Recife, PE, Brazil \\ ${ }^{\mathrm{d}}$ Department of Nuclear Energy, Federal University of Pernambuco, \\ CEP 50740-540 Recife, PE, Brazil
}

Received: April 11, 2010; Revised: May 21, 2010

\begin{abstract}
This work investigates the effect of particle size in the thermoluminescence (TL) response of a quartz crystal that was initially crushed and classified into ten size fractions between $38 \mu \mathrm{m}$ and $5 \mathrm{~mm}$. Aliquots of each size fraction were sensitized with a dose of $25 \mathrm{kGy}$ of $\gamma$ rays and heat-treatments at $400{ }^{\circ} \mathrm{C}$. TL glow curves of sensitized and non-sensitized samples were recorded as a function of different test-doses of $\gamma$ rays. For the non-sensitized samples, the TL peak near $325^{\circ} \mathrm{C}$ increases with the decrease in particle size. In the case of sensitized samples, a strong TL peak near $300{ }^{\circ} \mathrm{C}$ increases with the increase in particle size up to mean grain size equal to $304 \mu \mathrm{m}$. Above $304 \mu \mathrm{m}$, an abrupt reduction in the TL intensity is noticed for the sensitized peak. These effects are discussed in relation to the specific surface area of quartz particles and the intensity of the electron paramagnetic resonance signal of the $\mathrm{E}_{1}{ }_{1}$ center induced by the sensitization process.
\end{abstract}

Keywords: natural quartz, particle size, thermoluminescence, EPR spectroscopy

\section{Introduction}

Thermoluminescence (TL) of $\alpha$-quartz has been the subject of numerous investigations in the last five decades since the discovery of its practical use for archeological and geological dating. Detailed descriptions of TL in quartz including numerous references are available in review papers by McKeever ${ }^{1}$, Wintle ${ }^{2}$ and Preusser et al. ${ }^{3}$. TL glow curves of quartz grains usually found in sediments and rocks or even prepared from single crystals are composed by several peaks appearing near $110,170,320$ and $370{ }^{\circ} \mathrm{C}$. The peak near $110{ }^{\circ} \mathrm{C}$ usually shows a high sensitivity compared to the other glow peaks. Zimmerman ${ }^{4}$ was one of the first to report an increase in the sensitivity of this peak using $\gamma$ doses of few hundred Gy and heat treatment at $500-900^{\circ} \mathrm{C}$. This procedure was further studied and let the extensive use of the $110{ }^{\circ} \mathrm{C}$ peak for dating and environmental dosimetry ${ }^{5-8}$. However, the TL signal of the $110^{\circ} \mathrm{C}$ peak shows a fast anomalous fading at room temperature and due to this reason it is not used for TL dosimetry of $X$ and $\gamma$ rays $^{9-11}$. Until now, the peaks appearing near 320 and $370{ }^{\circ} \mathrm{C}$ have been mainly used in dating protocols ${ }^{2,3}$ but they are not used in TL dosimetry due to its low sensitivity. For instance, these peaks are not suitable for clinical dosimetry because for such kind of application the TL dosimeter (TLD) should assess a large range of doses from $10^{-6}$ to $10^{2} \mathrm{~Gy}^{12}$.

Recently, the possibility to produce a strong TL peak near $300^{\circ} \mathrm{C}$ in natural quartzes taken from different deposits was reported ${ }^{13-15}$ Combining high doses of $\gamma$ radiation and heat-treatments, it was possible to increase the TL sensitivity of a $280{ }^{\circ} \mathrm{C}$ peak more than 1000 times. Using this procedure, it became possible to assess doses as low as $10^{-4}$ Gy using discs with a $6 \mathrm{~mm}$ diameter manufactured from a quartz block taken from the Solonópole district located in the Ceará State of Brazil ${ }^{14}$. It was also observed that the outcome of sensitization was related to the impurity content ratios of aluminum $\left(\mathrm{Al}^{3+}\right)$, lithium $\left(\mathrm{Li}^{+}\right)$and hydroxyl $\left(\mathrm{OH}^{-}\right)$which exist as impurities into the quartz lattice and varies from one deposit to the other. Lattice impurities usually give rise to precursors of point defects that act as electron traps or recombination centres during ionizing radiation and TL output. In addition, it was reported that the dispersion involved with the TL output around $300{ }^{\circ} \mathrm{C}$ for samples prepared from the same crystal is supposedly attributable to local variation in $\mathrm{OH}^{-}, \mathrm{Al}^{3+}$ and $\mathrm{Li}^{+}$concentrations ${ }^{15}$. The heterogeneity in the spatial distribution of lattice impurities is a common feature in natural single crystals but it can be reduced by powdering and homogenization procedures ${ }^{16}$. In particular, the non-uniform distribution of TL intensities observed in a lot of TLDs prepared from single crystals can be minimized when the TLDs are prepared from particles resultant from the powdering of the crystal ${ }^{17}$.

As it is well known, Brazil has numerous natural deposits of raw quartz with different grades of optical quality and impurity content ${ }^{18}$. Looking for new applications of quartz resources, the sensitization process described above open the possibility to investigate the use of quartz particles in the production of TL dosimeters. Based on the detectable doses reported previously ${ }^{13-15}$, it seems that dosimeters prepared from sensitized quartz particles may be suitable for clinical (medical) and industrial applications ${ }^{17}$. If this issue is pursued, the 
first point to take into account is the effect of particle size in the TL response of the sensitized quartz. Many TLD materials are produced using powdering procedures and it is known that TL intensities are affected by the particle size ${ }^{17,19,20}$. However, few studies were carried out with the objective to understand the TL response of a given material as a function of its particle size $\mathrm{e}^{19-22}$. Thus, the aim of this study is to investigate the effect of particle size in the TL glow curves of natural quartz in non-sensitized and sensitized conditions.

\section{Experimental}

The natural quartz used in the present study was a single crystal extracted from one deposit located in the district of Solonópole (Ceará State, Brazil). Fragments of this crystal were cleaned with acetone for 15 minutes using an ultrasonic bath and then were manually crushed using an agate mortar and pestle. After crushing, the particles were classified into fine $(300 \times 425 \mu \mathrm{m}, 150 \times 315 \mu \mathrm{m}, 150 \times 300 \mu \mathrm{m}$, $75 \times 150 \mu \mathrm{m}, 38 \times 75 \mu \mathrm{m}$ and $<38 \mu \mathrm{m})$ and coarse fractions $(2.8 \times 4.8 \mathrm{~mm}, 2.0 \times 2.8 \mathrm{~mm}, 1.7 \times 2.0 \mathrm{~mm}$ and $0.85 \times 1.7 \mathrm{~mm})$ by using standard Tyler sieves. The particle size distributions and the specific surface area of the fine fractions were measured with a laser particle size analyzer, model Malvern Mastersizer 2000 with the Hydro 2000MU sample dispersion accessory. For these fractions, the mean particle size $\left(D_{\mathrm{m}}\right)$ corresponds to the aperture associated with $50 \%$ of passing material. For the coarse fractions, $D_{\mathrm{m}}$ corresponds to the average of the lengths measured along three directions of quartz grains with a digital microscope.

Aliquots of each size fraction were sensitized with a dose of $25 \mathrm{kGy}$ of ${ }^{60} \mathrm{Co}$ in a $\gamma$-cell irradiator with a dose rate close to $10 \mathrm{kGy} / \mathrm{h}$. After, three heat-treatments were successively performed in a muffle furnace as follows: continuous heating up to $400{ }^{\circ} \mathrm{C}$, annealing for one hour at $400{ }^{\circ} \mathrm{C}$, cooling, annealing for two hours at $100{ }^{\circ} \mathrm{C}$ and cooling. This thermal cycle completed the sensitization process and it was adopted as the standard annealing procedure throughout this work. For non-sensitized samples, this heat-treatment was also performed in order to remove the TL signals caused by previous exposure to natural radiation.

For the study of TL response as a function of particle size, three aliquots of $5 \mathrm{mg}$ were prepared for fine size fractions $(<425 \mu \mathrm{m})$. In the case of coarse particles $(>850 \mu \mathrm{m})$, the TL measurements were carried out in individual particles. The weight of each particle was ranged from 5 to $25 \mathrm{mg}$ according to its size. In order to compare with our previous data, the TL response was also read at three single crystals extracted from the same block. These samples are discs with $6 \times 1 \mathrm{~mm}^{2}$ which were sensitized with the same conditions described above. The non-sensitized samples were exposed to doses of $0.05,0.5$, 2 and $5 \mathrm{kGy}$ of ${ }^{60} \mathrm{Co}$ called here test-doses to distinguish them from the sensitization dose $(25 \mathrm{kGy})$. The sensitized samples were exposed to single test-doses of $50 \mathrm{mGy}$. The TL glow curves were recorded from 50 to $400{ }^{\circ} \mathrm{C}$ using a Harshaw 3500 reader with a heating rate equal to $2{ }^{\circ} \mathrm{Cs}^{-1}$. The TL signals of each aliquot were normalized into respect with the sample weight and test-dose. The mean TL intensities of the three measurements were obtained integrating the TL glow curves between 175 to $390{ }^{\circ} \mathrm{C}$.

In order to check the induction of lattice distortions in the crystal structure of quartz grains due to the severity of the manual crushing, standard X-ray diffraction (XRD) analyses were carried out in samples of very fine size fractions, i.e., $75 \times 150 \mu \mathrm{m}, 38 \times 75 \mu \mathrm{m}$ and $<38 \mu \mathrm{m}$. For this, XRD patterns were obtained with a $2 \theta$ diffractometer between 10 to $60^{\circ}$ using $\mathrm{Cu}-\mathrm{K} \alpha$ radiation. The full width at half maximum (FWHM) of the diffraction peaks related to $(10 \overline{1} 0),(10 \overline{1} 1)$ and $(11 \overline{2} 0)$ planes were measured. Afterwards, scanning electron microscopy (SEM) was also used to observe the shape and fracture patterns of the crushed particles classified in several size fractions.

The electron paramagnetic resonance (EPR) spectroscopy was carried for the fine size fractions $(<425 \mu \mathrm{m})$. The samples (200 mg) were placed into fused quartz tubes with inner diameter equal to $3 \mathrm{~mm}$. For each sample, three measurements were carried out in a Bruker EMX 10-Plus spectrometer operating at the X-band $(\sim 9.83 \mathrm{GHz})$ provided with a high sensitive cylindrical cavity. Initially, the magnetic field was swept from 1000 to $5500 \mathrm{G}$. Then, the EPR intensity was recorded at room temperature by sweeping the magnetic field from 3400 to $3530 \mathrm{G}$ setting the parameters as follows: modulation amplitude of the magnetic field: $1 \mathrm{G}$; modulation frequency: $100 \mathrm{kHz}$; time constant: $10.24 \mathrm{~ms}$; conversion time: $20 \mathrm{~ms}$; receiver gain: $1 \times 10^{4}$; number of scans: 10 . For specific magnetic field intervals, the signal was recorded as a function of the microwave power ranging from 0.002 to $63.25 \mathrm{~mW}$. The peak-to-peak intensities of EPR signals were measured in non saturated conditions.

\section{Results}

The TL curves of the non-sensitized samples with particle sizes ranging from 38 to $425 \mu \mathrm{m}$ show glow peaks near 90, 215 and $325^{\circ} \mathrm{C}$. An intense TL peak was observed at $90{ }^{\circ} \mathrm{C}$ that corresponds to the well-documented $110^{\circ} \mathrm{C}$ peak of quartz. In the present study, the peak at $90{ }^{\circ} \mathrm{C}$ was not considered due to its unstable behavior at room temperature. Figure 1a shows the characteristic TL curves for samples with different particle sizes irradiated with a test-dose of $50 \mathrm{~Gy}$. These results show that the TL intensity of the peak near $325^{\circ} \mathrm{C}$ increases with decreasing particle size. The TL glow curves of samples irradiated at $500 \mathrm{~Gy}$ are shown in Figure $1 \mathrm{~b}$. Besides the 110 and $325^{\circ} \mathrm{C}$ glow peaks, a new peak is observed near $215^{\circ} \mathrm{C}$. In this case, an opposite effect is observed with respect to particle size, i.e., the TL intensity at $215{ }^{\circ} \mathrm{C}$ decreases with decreasing particle size.

Figure 2 summarizes the behavior of the TL intensity integrated from 175 to $390{ }^{\circ} \mathrm{C}$ as a function of the mean particle size $\left(D_{\mathrm{m}}\right)$ for non-sensitized samples. In this figure, the TL intensity was normalized in relation to the intensity measured for the size fraction $<38 \mu \mathrm{m}$. According to Figure 2, the samples irradiated with 50 and 500 Gy show a similar behavior in relation to the mean particle size, i.e., the increase in TL intensity is observed for samples with $D_{\mathrm{m}}<150 \mu \mathrm{m}$. In case of samples irradiated at 2 and $5 \mathrm{kGy}$, the TL intensity is not clearly correlated with $D_{\mathrm{m}}$ due to the increase of the peak near $215^{\circ} \mathrm{C}$. As shown in Figure 1b, this peak increase with the increase in particle size. Thus, the increase of the $325^{\circ} \mathrm{C}$ peak with the decrease in particle size was completely surpassed by the increase of the $215^{\circ} \mathrm{C}$ peak.

In order to better observe the effect of particle size in the TL response, the glow curves of the sensitized samples were divided in two groups. Figure 3 a shows the TL glow curves for fine fractions and Figure $3 \mathrm{~b}$ shows the TL glow curves for coarse fractions. In Figure 3a, an increase in the TL intensity of the peak near $300^{\circ} \mathrm{C}$ is observed for larger particle sizes. This behavior is similar to that observed for the peak near $215^{\circ} \mathrm{C}$ occurring in non-sensitized samples (Figure 1b). In addition, it is observed that the TL peak near $300^{\circ} \mathrm{C}$ shifts to $280^{\circ} \mathrm{C}$ for particles classified as coarse fractions. In principle, it would be expected that this shift should be noticed in the opposite sense because it is believed that the temperature set is reached in a smaller amount of time when fine particles are distributed over the heating planchet of the TL reader. This statement is based on the assumption that the heating flow rate through a mass of insulating material is higher for fine grains. In this way, the onset of the luminescence should occur at lower temperature for fine grains. Therefore, the temperature shift shown in Figures $3 a$ and $b$ cannot be explained by the effect of the 


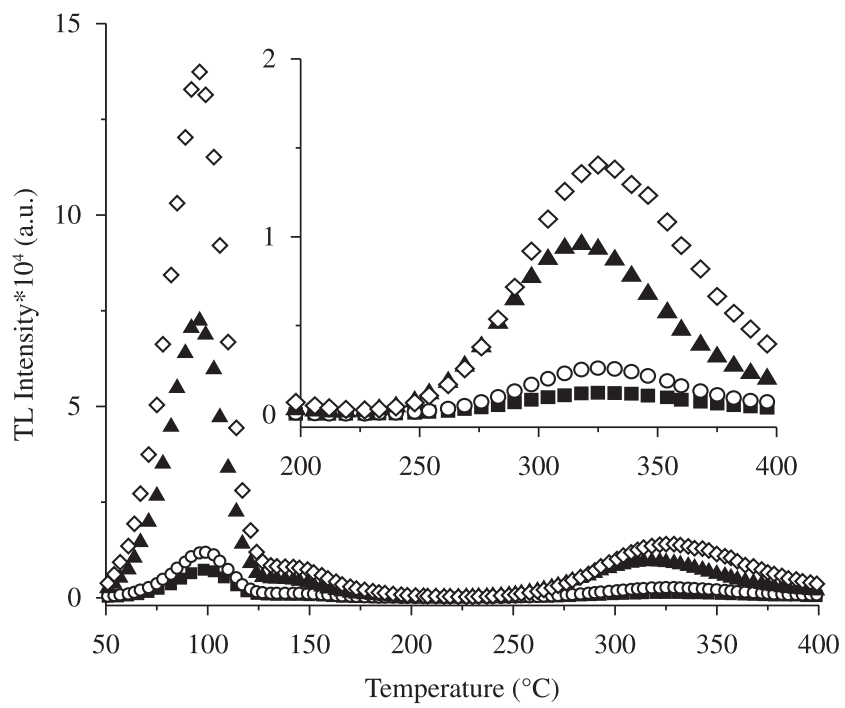

(a) 50 Gy

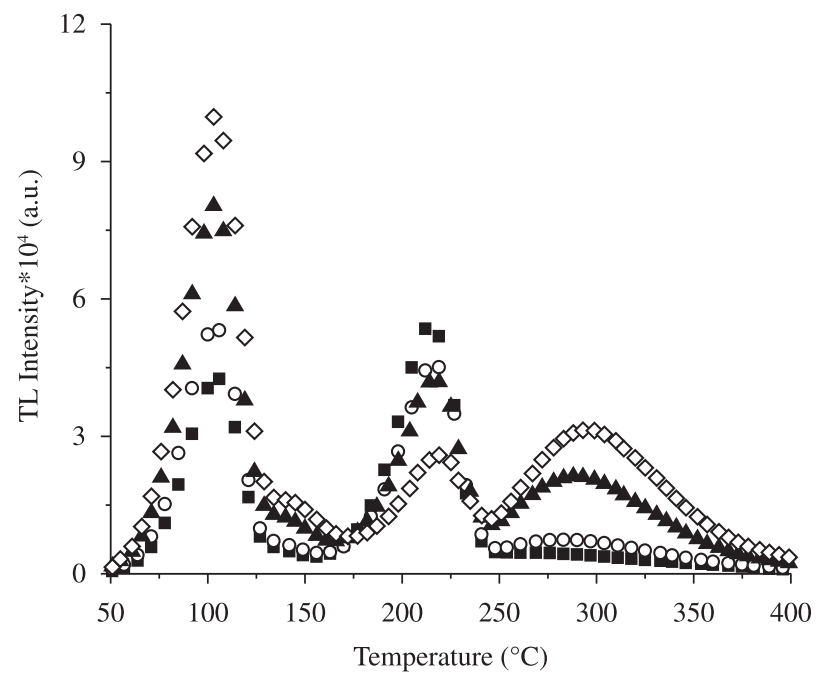

(b) $500 \mathrm{~Gy}$

$300 \times 425 \mu \mathrm{m} \quad \circ 75 \times 150 \mu \mathrm{m} \quad \Delta 38 \times 75 \mu \mathrm{m} \quad \diamond<38 \mu \mathrm{m}$

Figure 1. TL glow curves of quartz particles exposed to different test-doses of $\gamma$ radiation.

particle size on the heat flow during the TL readings. Certainly, the temperature shift of the second peak is connected with the sensitization of a broad TL peak occurring between 200 and $300{ }^{\circ} \mathrm{C}$ when quartz is treated with high $\gamma$ dose and heat. In addition, Figure 3 shows that the peak at $90{ }^{\circ} \mathrm{C}$ shows different intensities that can be explained by the anomalous fading ${ }^{9-11}$, which occurs during the interval of TL readings. On the other hand, the intensity of the TL peak near $300^{\circ} \mathrm{C}$ seems to be very stable as a function of time.

Figure 4 shows the TL signals integrated from 175 to $390{ }^{\circ} \mathrm{C}$ as a function of the mean particle size for sensitized quartz samples. It can be seen that the TL intensity increases as the particle size increases from $D_{\mathrm{m}}=18$ to $D_{\mathrm{m}}=304 \mu \mathrm{m}$. For particles larger than $304 \mu \mathrm{m}$, an abrupt decrease in the TL intensity is noticed and no important change in TL intensity is observed for particle sizes larger than $2 \mathrm{~mm}$. In this figure, the sample with the largest $D_{\mathrm{m}}$ is a solid disc with diameter of 6 and $1 \mathrm{~mm}$ thick. This disc was prepared directly from the crystal plate and it was not submitted to the crushing procedure.

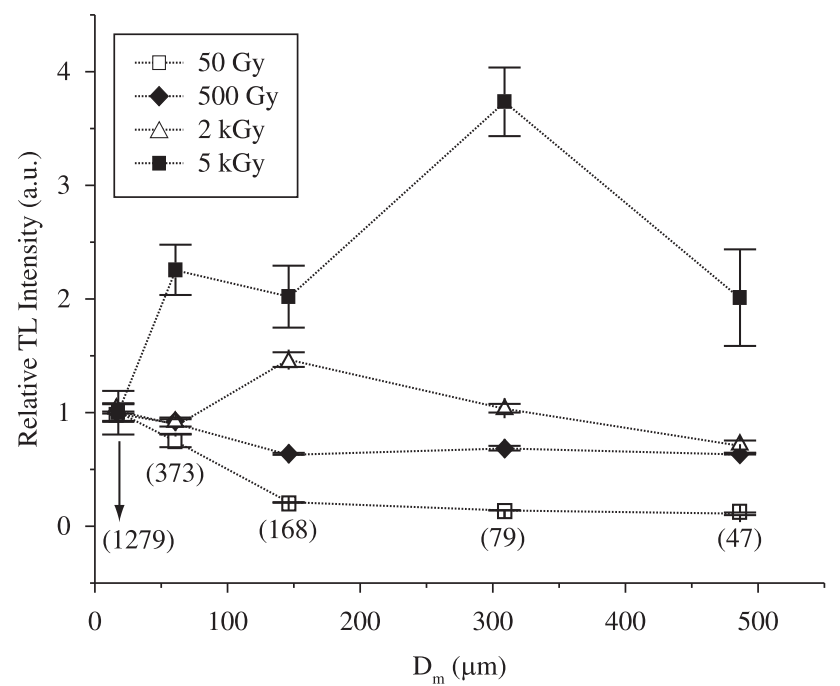

Figure 2. Relationship between TL intensity integrated from 175 to $390{ }^{\circ} \mathrm{C}$ and the mean particle size $\left(D_{\text {m }}\right)$ for non-sensitized quartz. Values between parentheses are the specific surface area measured in $\mathrm{cm}^{2} \mathrm{~g}^{-1}$.

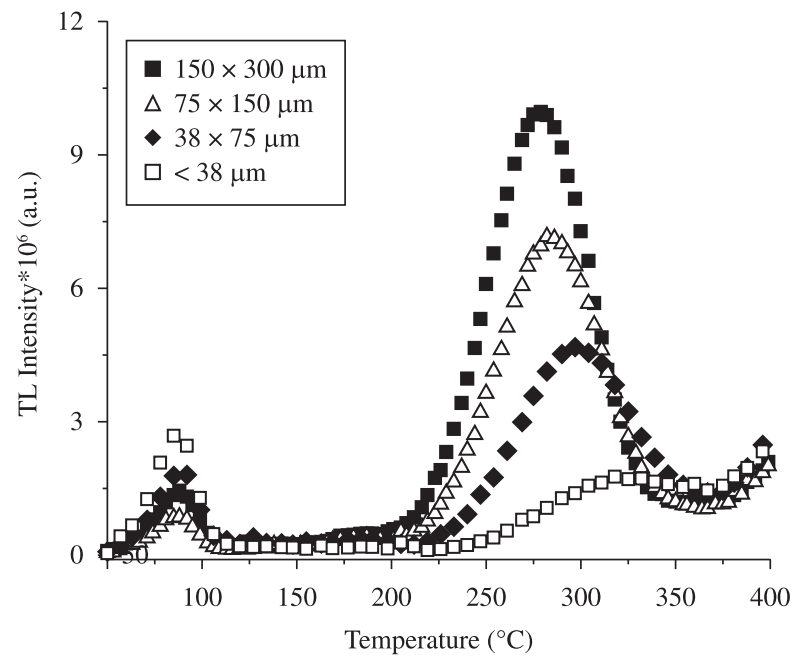

(a)

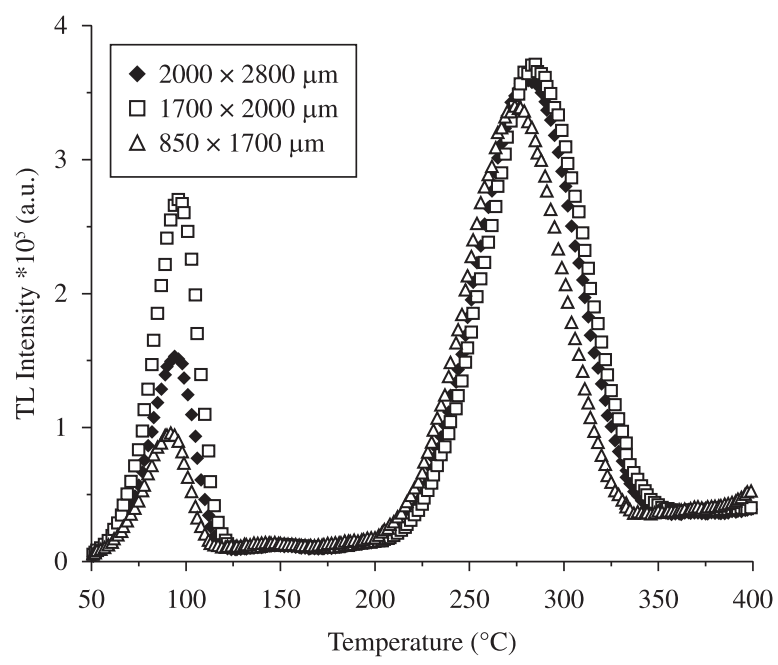

(b)

Figure 3. TL glow curve of quartz particles sensitized with $25 \mathrm{kGy}$ of $\gamma$ radiation and heat-treatments at $400{ }^{\circ} \mathrm{C}$ (test dose: $50 \mathrm{mGy}$ ). 


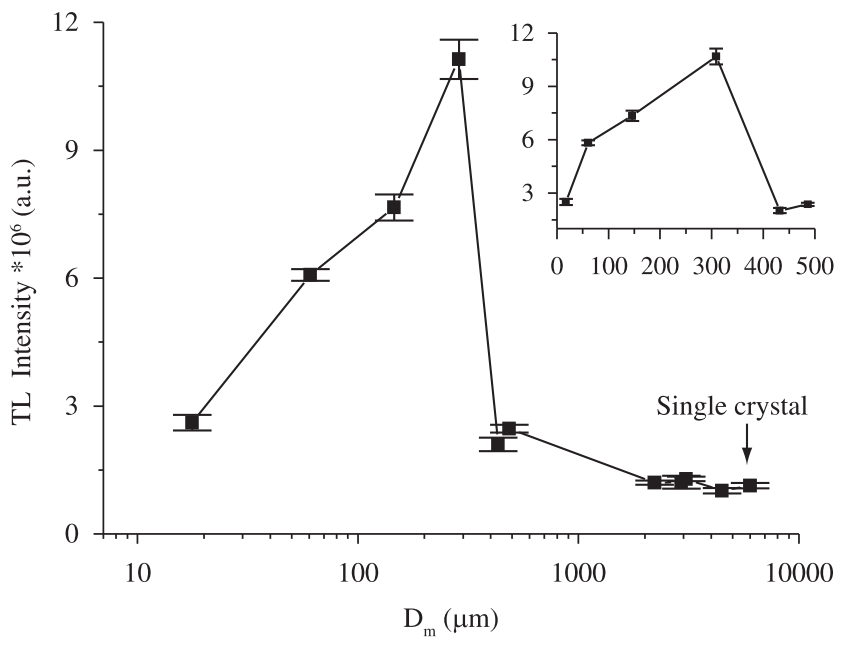

Figure 4. Relationship between TL intensity integrated from 175 to $390{ }^{\circ} \mathrm{C}$ and the mean particle size $\left(D_{\mathrm{m}}\right)$ for sensitized quartz (test dose: $50 \mathrm{mGy}$ ). The inset plot corresponds to the initial part of the figure in a linear scale.

These measurements suggest a reliable similarity between the TL responses of coarse particle sizes and the TL response of a single crystal prepared from the same sensitized quartz.

\section{Discussion}

Previous studies showed that the manual crushing of quartz particles with mortar and pestle can induce mechanical damage or amorphization into the crystalline structure when fine grains are submitted to high pressures ${ }^{23-25}$. However, no change in the width of the $(10 \overline{1} 0),(10 \overline{1} 1)$ and $(1 \overline{1} 0)$ diffracting peaks was observed with the FWHM analysis carried out in $75 \times 150 \mu \mathrm{m}, 38 \times 75 \mu \mathrm{m}$ and $<38 \mu \mathrm{m}$ size fractions. Based on this analysis, the assumption that the decreasing TL intensity for $D_{\mathrm{m}}<304 \mu \mathrm{m}$ shown in Figure 4 would be associated with amorphization or mechanical damage into the crystalline structure of quartz grains can be ruled out. Similarly, the SEM analysis did not reveal any substantial difference in the morphology of the quartz particles. For instance, Figure 5 shows typical images obtained for particles with $\mathrm{D}_{\mathrm{m}}$ equal to 304 and $486 \mu \mathrm{m}$. Besides the size range and the presence of very fine grains deposited on the surface of $304 \mu \mathrm{m}$ particles, no difference is observed in fracture patterns that could be associated with the abrupt change of TL intensity shown in the Figure 4. It is observed that brittle micro-cracking is the principal mechanism of size reduction related to the crushing procedure performed here.

The effect of the particle size in the TL response of non-sensitized quartz can be explained by the increase in the specific surface area for fine quartz grains. For test-doses 50 and $500 \mathrm{~Gy}$, it was observed that the TL intensity increases with decreasing particle size when $D_{\mathrm{m}}<150 \mu \mathrm{m}$. As shown in Figure 2, the specific surface area for size fractions $<38 \mu \mathrm{m}$ and $38 \times 75 \mu \mathrm{m}$ are larger than the others. A similar behavior was previously reported for particles of potassium iodate (KI) and amorphous silica exposed to similar test-doses ${ }^{21,22}$. As stated before, the TL output increases with decreasing particle size because a much higher surface area of material is exposed during the TL reading. The effect of the specific surface area on the TL intensity was not observed when test doses such as 2 and $5 \mathrm{kGy}$ were administered to quartz grains due to the onset of the peak near $215^{\circ} \mathrm{C}$. As shown in Figure $1 \mathrm{~b}$, this peak increases with the increase in particle size and this

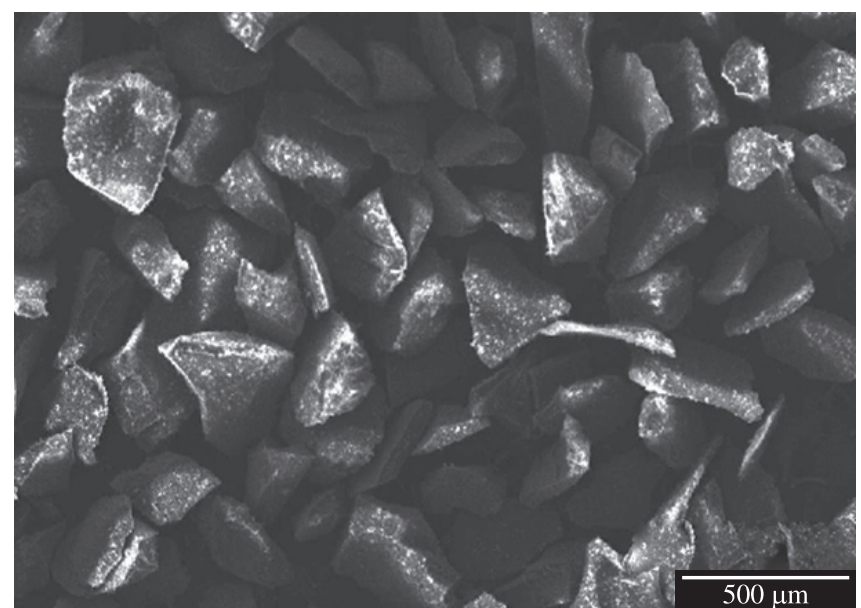

(a) $D_{m}=304 \mathrm{~mm}$

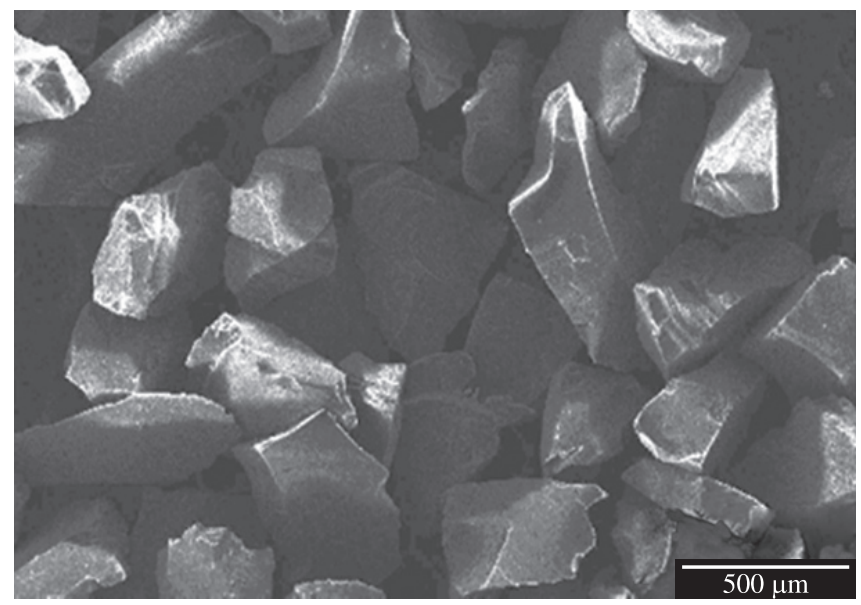

(b) $D_{m}=486 \mathrm{~mm}$

Figure 5. SEM micrographs of quartz particles with different mean grain sizes.

effect is probably associated with the beginning of the sensitization process responsible for the strong peak near $300^{\circ} \mathrm{C}$.

For sensitized samples, the small increase in TL intensity observed in the right side of Figure 4, when TL intensities of the single crystal and fragments of few millimeters are compared with that of particles with $D_{\mathrm{m}} \approx 450 \mu \mathrm{m}$, can also be attributed to the increase of the specific surface area. In the sequence, the abrupt increase in TL response for $D_{\mathrm{m}}=304 \mu \mathrm{m}$ and the subsequent decrease noticed for finer particles cannot be merely explained by the effect of specific surface area. As a first approximation, these results can be explained by the effect of particle size during the irradiation of quartz with high dose of $\gamma$ radiation. For some reason that is not clear, the sensitization with $25 \mathrm{kGy}$ is more effective to create paramagnetic centers acting as electron traps and recombination centers in those particles with $D_{\mathrm{m}}=304 \mu \mathrm{m}$. It is believed that finer particles do not absorb the radiation in the same way as particles with grain size larger than $304 \mu \mathrm{m}$. Thus, the $280{ }^{\circ} \mathrm{C}$ TL peak noticed for quartz particles with $D_{\mathrm{m}}=304 \mu \mathrm{m}$ could be explained by the concomitant effects of the absorption of high $\gamma$ dose and the specific surface area of the particles within this size range. In order to examine this hypothesis in more detail, the fine size fractions of sensitized quartz were further investigated with EPR spectroscopy and the most important results are described below. 
The EPR spectra shown in Figure 6 illustrate typical derivative lines of the EPR signals recorded for the fine size fractions of quartz particles measured before and after the sensitization process. In the Figure $6 \mathrm{a}$, the EPR lines observed in the region between 3400 and $3520 \mathrm{G}$, characterized by the $\mathrm{g}$ factors at $2.0496,2.0072$ and 2.0037 , were assigned to paramagnetic centers related to silicon vacancies. The existence of silicon vacancies in the quartz structure is expected to lead to hole trapping by two oxygen ions with the formation of various $\mathrm{O}_{2}{ }^{3-}$ centers. In this case, the values of the $g$ factors mentioned above corresponds to the presence of $\mathrm{O}_{2}{ }^{3-} / \mathrm{M}^{+}$centers (where $\mathrm{M}^{+}=\mathrm{Li}^{+}, \mathrm{Na}^{+}$) in the quartz structure ${ }^{26-28}$. It was reported that these centers are stable at room temperature and can be observed up to $350{ }^{\circ} \mathrm{C}^{26,28}$. Because the sample of Figure $6 \mathrm{a}$ was not irradiated, it is suggested that the Si-vacancy centers observed in Solonópole quartz were originally created during the crushing procedure but the intensity of the EPR lines of these centers remarkably increased after the sensitization with $25 \mathrm{kGy}$ and heat-treatment at $400{ }^{\circ} \mathrm{C}$, as can be seen in Figure $6 \mathrm{c}$.

As shown in Figure 6b, no EPR signals were observed for non sensitized samples in the field range of 3508 and $3525 \mathrm{G}$. In case of sensitized samples, definite EPR signals appeared in this field range for all size fractions that was investigated. As shown in Figure 6d, these signals, characterized by g values equal to 2.0012, 1.9950 and 1.9940 , were previously detected in synthetic quartz crystals doped with germanium. These signals were assigned to $\mathrm{E}{ }_{1}$ centers perturbed by a $\mathrm{Ge}^{4+}$ substituting for $\mathrm{Si}^{4+}$ in the $\mathrm{SiO}_{4}$ tetrahedron ${ }^{29,30}$. The accepted

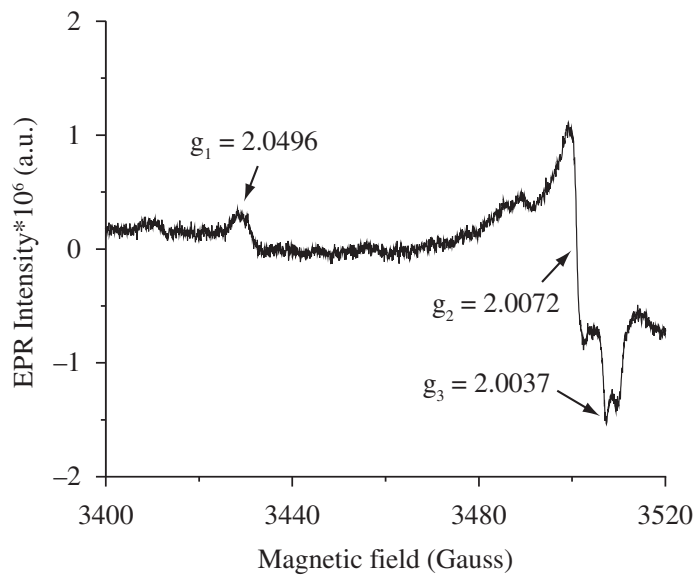

(a)

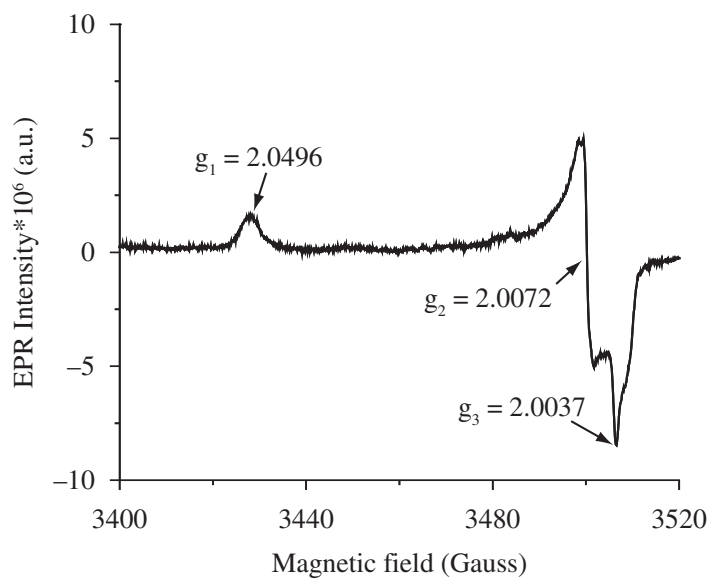

(c) model for the E', center corresponds to an oxygen vacancy with an unpaired electron in the $\mathrm{sp}^{3}$ hybrid orbital extending into the vacancy from an adjacent $\mathrm{Si}$ atom ${ }^{30,31}$.

The relationship between the intensity of the central signal $\left(\mathrm{g}_{2}=1.9950\right)$ related to $\mathrm{E}^{\prime}$, center and $D_{\mathrm{m}}$ is shown in Figure 7. It is observed that the intensity of this signal initially increases and then decreases with the increase in $D_{\mathrm{m}}$. It is also observed that the size fractions with $D_{\mathrm{m}}$ equal to 138 and $304 \mu \mathrm{m}$ show similar EPR intensity values for this defect centre. Compared to the TL response shown in the inset plot of Figure 4, one observes that the decrease in both signals (TL and EPR) occurs for particles with similar sizes. Previously, several authors suggested that paramagnetic centers of the E' family play the role of electron traps during quartz irradiation ${ }^{32-34}$. Thus, the results of the present study let us to suggest that the formation of $\mathrm{E}_{1}{ }_{1}$ centers (perturbed by substitutional Ge species) by the sensitization process described above are dependent of the particle size and help to explain the relationship observed between the TL response of the $280^{\circ} \mathrm{C}$ glow peak of Solonópole quartz and its particle size. Using a similar attempt to correlate the $\mathrm{g}_{2}$ value of Si-vacancy centers, no clear relationship was found between this signal and the TL response of the sensitized peak. Further investigations are required to examine the effect of particle size in the creation of paramagnetic centers that act as recombination centers during the TL output such as the $\left[\mathrm{AlO}_{4}\right]^{0}$ hole-centers. The EPR signals of this defect can be only detected at $100 \mathrm{~K}$ or at lower temperatures ${ }^{1,31}$.

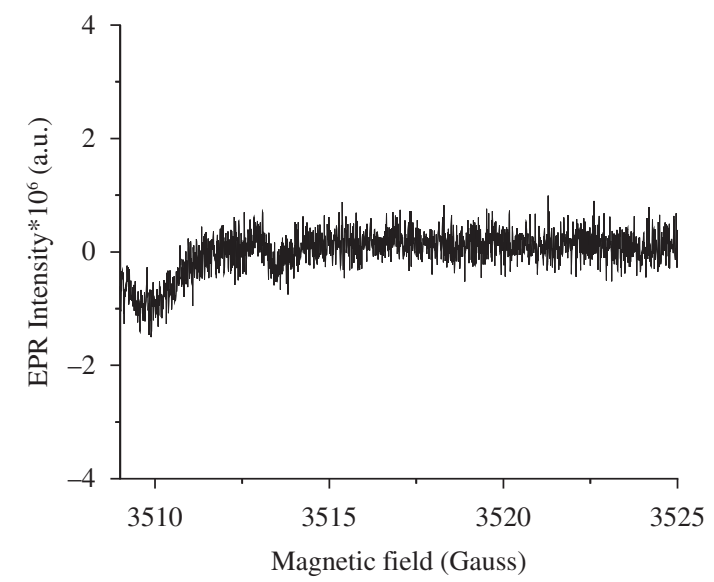

(b)

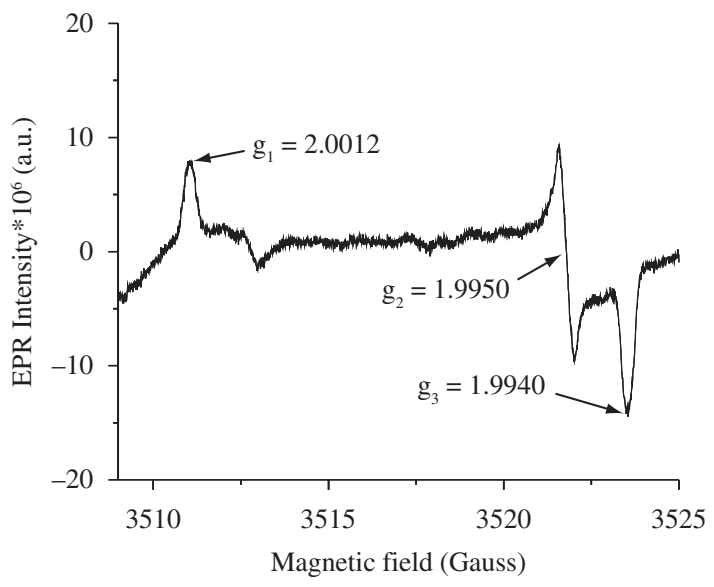

(d)

Figure 6. EPR spectra of quartz particles with $D_{\mathrm{m}}=138 \mu \mathrm{m}$ obtained at room temperature in non-sensitized (a, b) and sensitized (c, d) conditions. 


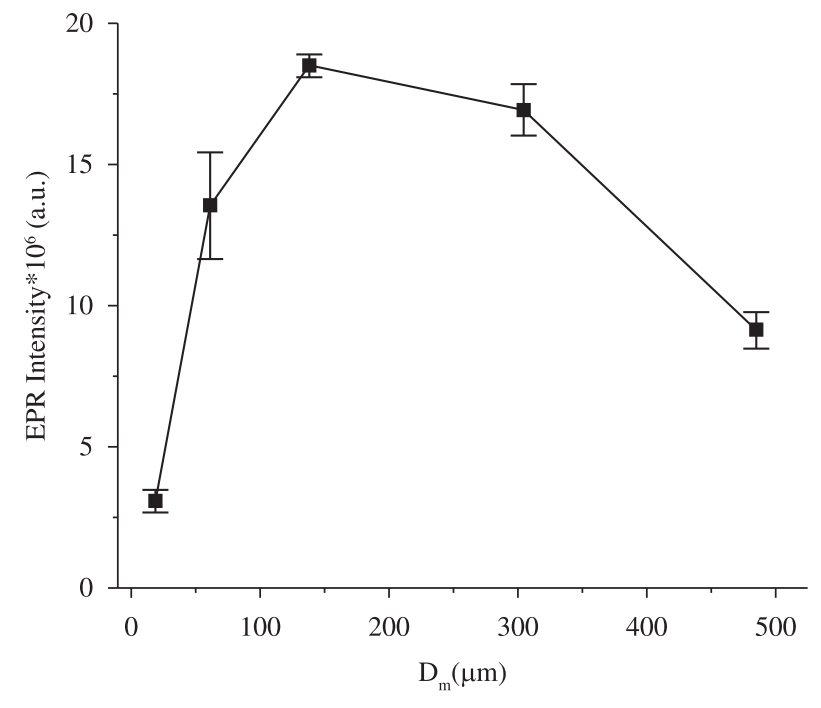

Figure 7. Relationship between the EPR intensity of $E^{\prime}$ centers perturbed by substitutional Ge species and the mean particle size $\left(D_{\mathrm{m}}\right)$ of sensitized quartz.

\section{Conclusion}

For non-sensitized natural quartz extracted from the Solonópole district, the increase in TL intensity integrated from 175 to $390{ }^{\circ} \mathrm{C}$ with particle size decreasing is associated with the increase in the peak near $325^{\circ} \mathrm{C}$ and this result is explained by the increase in the specific surface area. In this study, this effect was clearly observed using test-doses ranging from 50 to $500 \mathrm{~Gy}$. For test-doses in the range of a few kGy, the effect of the specific surface area is not observed due to the onset of an additional peak near $215^{\circ} \mathrm{C}$, which showed an opposite behavior with particle size. After sensitization with $25 \mathrm{kGy}$ and $400{ }^{\circ} \mathrm{C}$, the effect of the specific surface area is noticed only when the TL intensities of fragments of a few millimeters are compared to those particles with mean grain size close to $300 \mu \mathrm{m}$. The subsequent decrease in TL intensity observed for particles with mean grain size $<300 \mu \mathrm{m}$ can be explained by the inferior number in E', centers perturbed by substitutional Ge created during the sensitization process, which probably act as electron traps during irradiation with test-doses. This work led to the conclusion that Solonópole quartz with mean particle size close to $300 \mu \mathrm{m}$ has suitable properties for future use in TL dosimetry.

\section{Acknowledgments}

The authors are thankful to Profa ${ }^{a}$. Márcia Carvalho A. Fantini (IF-USP, São Paulo) for XRD analyses. One the authors (ABCJr.) is grateful to CAPES for the scholarship provided. This work was supported by CNPq and FINEP/INFRA-2005 (Brazil).

\section{References}

1. McKeever SWS. Thermoluminescence in quartz and silica. Radiation Protection Dosimetry. 1984; 8(1-2):81-98.

2. Wintle AG. Luminescence dating: laboratory procedures and protocols. Radiation Measurements. 1997; 27(5-6):769-817.

3. Preusser F, Chinthambo ML, Gotte T, Martini M, Ramseyer K, Sendera EJ et al. Quartz as a natural luminescence dosimeter. Earth-Science Reviews. 2009; 97(1-4):196-226.

4. Zimmerman J. The radiation-induced increase of the $100{ }^{\circ} \mathrm{C}$ thermoluminescence sensitivity of fired quartz. Journal of Physics C: Solid State Physics. 1971; 4:3265-3276.
5. Fleming SJ. Pre-dose technique - new thermoluminescent dating method. Archaeometry. 1973; 15:13-30.

6. Bailiff IK and Haskell EH. The use of pre-dose technique for environmental dosimetry. Radiation Protection Dosimetry. 1983; 6(1-4):245-248.

7. Yang XH and McKeever SWS. The pre-dose effect in crystalline quartz. Journal of Physics D: Applied Physics. 1990; 23(2):237-244.

8. Benny PG, Sanjeev N, Gundu Rao T K and Bhatt BC. Gamma ray induced sensitization of $100^{\circ} \mathrm{C}$ TL peak in quartz separated from sand. Radiation Measurements. 2000; 32(3):247-252.

9. David M and Sunta CM. Quartz dosimeter for archaeological dating. Nuclear Tracks. 1985; 10(4-6):625-629.

10. Ehlermann DE and Khan HM. Analysis of fading characteristics of quartz sand as a dosimeter and in irradiation identification applications. Radiation Physics and Chemistry. 1985; 46:1185-1187.

11. Yazici AN and Topaksu M. The analysis of thermoluminescence glow peaks of unannealed synthetic quartz. Journal of Physics D: Applied Physics Radiation. 2003; 36(6):620-627.

12. Bos AJJ. High sensitivity thermoluminescence dosimetry. Nuclear Instruments and Methods in Physics Research B: Beam Interactions with Materials and Atoms. 2001; 184(1-2):3-28.

13. Khoury HJ, Guzzo PL, Barreto SB and Hazin CA. Effect of high gamma doses on the sensitisation of natural quartz used for thermoluminescence dosimetry. Radiation Effects \& Defects in Solids. 2007; 162(2):101-107.

14. Khoury HJ, Guzzo PL, Souza LBF, Farias TMB and Watanabe STL. Dosimetry of natural quartz sensitized by heat-treatment and high dose irradiation. Radiation Measurements. 2008; 43(2-6):487-491.

15. Guzzo PL, Khoury HJ, Miranda MR, Barreto SB and Shinohara AH. Point defects and pre-dose requirements for sensitization of the $300{ }^{\circ} \mathrm{C}$ TL peak in natural quartz. Physics and Chemistry of Minerals. 2009; 36(2):75-85.

16. Altree-Williams S, Byrnes JG and Jordan B. Amorphous surface and quantitative X-ray powder diffractometry. Analyst. 1981; 106(1258):69-75.

17. Mahesh K, Weng PS and Furetta C. Thermoluminescence in solids and its applications. Ashford: Nuclear Technology Publishing; 1989.

18. Iwasaki H, Iwasaki F, Oliveira VAR, Hummel DAC, Pasquali MA, Guzzo PL et al. Impurity content characterization of Brazilian quartz lascas. Japanese Journal of Applied Physics. 1991; 30(7):1489-1495.

19. Driscoll CMH and McKinlay AF. Particle size effects in thermoluminescent lithium fluoride. Physics in Medicine and Biology. 1981; 26(2):321-327.

20. Carlson GA and Lorence L. Particle size effect in CaF2: Mn/Teflon TLD response at photons energies from 5-1250 keV. IEEE Transactions on Nuclear Science. 1990; 37(5):1560-1563.

21. Dhoble SJ, Sahare PD and Moharil SV. Thermoluminescence and colour centres in KI: particle size effect. Journal of Physics: Condensed Matter. 1991;(9):1189-1191.

22. Ranjbar AH, Durrani SA and Randle K. Electron spin resonance and thermoluminescence in powder form of clear fused quartz: effect of grinding. Radiation Measurements. 1999; 30(1):73-81.

23. Hazen RM, Finger LW, Hemley RJ and Mao HK. High-pressure crystal chemistry and amorphization of $\alpha$-quartz. Solid State Communications. 1989; 72(5):507-511.

24. Kingma KJ, Meade C, Hemley RJ, Mao H and Veblen DR. Microstructural observations of $\alpha$-quarz amorphization. Science. 1993; 259(5095):666-669.

25. Surinder MS and Sikka, SK. Pressure induced amorphization of materials. Progress in Materials Science. 1996; 40(1):1-11.

26. Mashkovtsev RI, Scherbakova MY A and Solitsev VP. EPR of radiation oxygen hole centres in $\alpha$-quartz. Tr. Inst. Geol. Geofiz. Transactions of Institute of Geolgy-Geophyiscs 1978; 385:78-86. 
27. Marfunin AS. Spectroscopy, luminescence and radiations centers in minerals. New York: Springer-Verlag; 1979.

28. Pan YM, Botis $S$ and Nokhrin S. Application of natural radiation-induced paramagnetic defects in quartz to exploration in sedimentary Basins. Journal of China University of Geosciences. 2006; 17(3):258-271.

29. Feigl FJ and Anderson JH. Defects in crystalline quartz: electron paramagnetic resonance of E' vacancy centers associated with germanium impurities. Journal of Physics and Chemistry of Solids. 1970; 31(4):575-596.

30. Ikeya M. New applications of electron spin resonance: dating, dosimetry and microscopy. World Scientific Publishing; 1993.
31. Halliburton LE. Defect models and radiation damage mechanisms in alpha-quartz. Crystal Lattice Defects and Amorphus Materials. 1985; 12(1-2):163-190.

32. Jani MG, Bossoli RB and Halliburton, LE. Further characterization of the $\mathrm{E}_{1}$ 'center in crystalline $\mathrm{SiO}_{2}$. Physical Review B. 1983; 27(4):2285-2293.

33. Jani MG, Halliburton, LE and Kohnke EE. Point defects in crystalline $\mathrm{SiO}_{2}$ : Thermally stimulated luminescence above room temperature. Journal of Applied Physics. 1983; 54(11):6321-6328.

34. Benny PG, Gundu Rao TK and Bhatt BC. The E' ${ }_{1}$-centre and its role in TL sensitization in quartz. Radiation Measurements. 2002; 35(4):369-373. 